\title{
Padrões de uso do smartphone potencialmente disfuncionais em adultos
}

\author{
portugueses
}

\section{Potentially dysfunctional patterns of smartphone use in Portuguese adults}

\author{
Isabel Silva*, Zélia Teixeira*, Margarida Soliz** \\ *UFP/Hospital-Escola da UFP/ Centro de Investigação FPB2S/APASD, **ARS Norte IP
}

\begin{abstract}
Resumo
A globalização da informação em múltiplos domínios da vida dos indivíduos congrega vantagens e riscos. $\mathrm{O}$ presente estudo propôs-se avaliar a existência de padrões de uso do smartphone potencialmente disfuncionais junto de uma amostra de conveniência constituída por 164 participantes adultos. Os participantes responderam a: versão portuguesa do Smartphone Addiction Inventory; questionário sociodemográfico; questionário de sintomas clínicos sugestivos de uso problemático/dependência de smartphones. Ainda que a maioria dos participantes (90.9\%) não apresente sintomas de perturbação associada ao uso de smartphones, $6.7 \%$ apresenta sintomas moderados e $2.4 \%$ sintomas graves. Os resultados sugerem a importância da realização de rastreios neste domínio. Palavras-chave: adição; smartphone; compulsão; tolerância; abstinência.
\end{abstract}

\begin{abstract}
The globalization of information in multiple domains of individuals' lives is associated to advantages, but also to risks. The present study aimed to evaluate the existence of potentially dysfunctional smartphone use patterns in a convenience sample of 164 adults. Participants answered to: Portuguese version of the Smartphone Addiction Inventory; Sociodemographic questionnaire; Questionnaire of clinical symptoms suggestive of problematic use/dependence on smartphones. Although majority of participants (90.9\%) showed no symptoms of disturbance associated with the use of smartphones, $6.7 \%$ presented moderate symptoms and $2.4 \%$ severe symptoms. These results underline the importance of screening in this domain.

Keywords: addiction, smartphone; compulsion, withdrawal, tolerance
\end{abstract}

$\mathrm{Na}$ era das tecnologias da comunicação e da informação (TCI) assistimos à reconfiguração, nos últimos 15 anos, de estilos de vida, formas de relacionamento, meios de trabalho, acesso ao conhecimento, entre muitas outras vertentes cruciais da existência humana. A informação, particularmente aquela que se sustenta das e nas novas tecnologias assumiu um papel indispensável na vida quotidiana, (Salehan, \& Negahban, 2013), e tem vindo a associar-se a um número crescente de actividades humanas.

O número de utilizadores de internet a nível mundial encontrava-se em 2015 nos 3.2 Mil Milhões, segundo um relatório emitido pela International Telecommunication Union (ITU, 2015). Este valor consistia na altura em $43 \%$ da população mundial e multiplicava por sete o número de utilizadores de internet no planeta no ano 2000 (que correspondia a 7\% da população global). Em 31 de março de 2017 encontrámos o valor de 3.739.698.500 utilizadores da internet em todo o mundo (49,7\% da população mundial), estando $17 \%$ destes valores localizados na Europa, com uma taxa de penetração na população de $77.4 \%$, e correspondendo a 636.791.824 indivíduos (Internet World Stats, 2017).

Temos, pois, as TCI como marcos incontornáveis das sociedades de hoje para o que contribuem um conjunto de factores que Pinna et al. (2015) enumeram: acessibilidade, disponibilidade, intimidade, alta estimulação e anonimato.

Paralelamente, a exuberância da vida contemporânea tem visto surgir vários comportamentos, para além dos associados tradicionalmente à ingestão de substâncias psicoativas, que produzem uma resposta de recompensa cerebral a curto prazo, que podem gerar comportamentos repetidos e persistentes apesar do conhecimento das suas consequências adversas, e que apresentam como expressão regular o controle diminuído sobre o comportamento por parte do indivíduo. Dadas as suas características específicas estas condições foram abordadas historicamente sob diferentes perspetivas, que vão desde a sua consideração no espectro impulsivo-compulsivo, à sua inserção no grupo das perturbações de controle dos impulsos e, mais recentemente, à sua inclusão na categoria das adições sem substâncias (Grant, Potenza, Weinstein, \& Gorelick, 2010).

Esta evolução espelha-se nas reflexões suscitadas pelas classificações internacionais, que no caso explicito da DSM-5, cria uma categoria para a adição ao jogo (retirando-a do grupo das perturbações do controle dos impulsos), e deixa em suspenso a abertura na próxima edição a outras perturbações semelhantes eventualmente a serem consideradas na secção Perturbações Relacionadas com Substâncias e Perturbações Aditivas que acolheu a perturbação de Jogo (A.P.A., 2013). Esta mudança, tardia para uns, e precoce para outros (Holden, 2010) reforçou a tendência para que o conceito de adição 
possa ser hoje aplicado na descrição de um conjunto heterogéneo de síndromes denominadas como adições comportamentais, novas adições, ou adições sem substâncias. As adições comportamentais podem ser definidas como desordens psicopatológicas emergentes, envoltas num crescente interesse científico e com significativas implicações sociais, culturais e económicas (Pinna, et al., 2015).

Um número crescente de estudos empíricos e de reflexões teóricas espelham a necessidade de clarificar o conceito de adições comportamentais, sendo certo que uma das perspetivas que sobressai é aquela que aponta um conjunto de fatores comuns entre as adições sem substâncias e as tradicionais adições químicas (Orford, 2008). Grant, Potenza, Weinstein, e Gorelick (2010), na mesma linha, organizam as semelhanças entre os dois tipos de adições em 7 áreas: história natural (percurso evolutivo do problema), fenomenologia (expressão da experiência aditiva, com forte desejo subjetivo, alteração da consciência semelhante à intoxicação, e sintomas de privação), tolerância, comorbilidade, sobreposição de contribuição genética, mecanismos neurobiológicos subjacentes (funções dos sistemas glutamatérgico, opióide, seretoninérgico e dopaminérgico) e resposta ao tratamento. Echeburrua e Corral (2010) referem-se aos comportamentos aditivos como uma patologia da liberdade, em especial quando refletem sobre o impacto das TIC enquanto actividades aparentemente inofensivas, banalizadas e indutoras de prazer, mas que ativam um conjunto de sistemas cerebrais predominantemente localizados no sistema límbico que têm um potencial interessante para criar dependência.

De entre os vários comportamentos que podem constituir-se como uma adição salientam-se o jogo (nas duas dimensões gaming e gambling), as compras, o sexo, a comida, a internet, o exercício físico, numa lista que recentemente se abriu por exemplo às redes sociais (Pinna et al., 2015; Rosemberg, \& Feder, 2014)

Já em 1996, Griffiths definia as adições tecnológicas como adições comportamentais (não químicas) que se sustentam na interação homem-máquina, podendo ser passivas (e.g. televisão) ou ativas (e.g. jogos e computador). Para o autor estes comportamentos induzem e reforçam efeitos que podem contribuir para o desenvolvimento de tendências aditivas. Para Pinna et al. (2015), as adições tecnológicas envolvem, na sua maioria, a internet, telefones móveis e videojogos, acrescentam à classificação acima referida o facto deste tipo de adições sustentadas nas TCI não terem que ser mutuamente exclusivas e definem-nas como detentoras de características que podem levar a que sejam igualmente consideradas em outros domínios aditivos, dando como exemplo a experiência de adição ao sexo, operacionalizada através do uso do telefone móvel. Esta associação dos telemóveis a comportamentos aditivos parece coincidir com o aparecimento relativamente recente do smartphone, que rapidamente destronou computadores tradicionais e portáteis, tornando-se a forma mais prática de aceder à Internet (Roberts, Yaia, Manolis, 2014). O smartphone, é o tema da adição tecnológica mais recente. Fruto de uma evolução tecnológica muito rápida, de um decréscimo relativo dos preços e da expansão das suas funções este dispositivo tornou-se um fenómeno global (Oulasvirta et al., 2012).

Hoje, o smartphone condensa um conjunto de aplicações e utilidades que o transformaram num computador de reduzidas dimensões, que permite uma utilização agregada não só de diferentes formas de comunicação (email, texting, redes sociais...) como também de múltiplas aplicações consideradas indispensáveis à vida humana na contemporaneidade. É na ultrapassagem dos limites (difíceis de estabelecer) da funcionalidade para a disfuncionalidade das novas tecnologias que se encastra a pertinência do presente estudo, pois se é certo que a tecnologia é uma facilitador da vida humana, também o é que pode resultar num uso inapropriado que em última instância redunda em adição (Turel, Serenko, \& Giles, 2011).

Este florescimento no uso do smartphone tornou mais saliente a temática de podermos estar perante uma adição, em que se verifica a passagem de um hábito para uma necessidade (Kwon, Kim, Cho, \& Yang, 2013; Roberts, Yaya, Manolis, 2014) ou a passagem do gostar para o querer usar o smartphone, ponto de inflexão considerado por Grover et al. (2011) num processo que é progressivo, frequentemente iniciado com usos aparentemente benignos deste dispositivo, que são moldados por factores múltiplos até configurarem uma padrão aditivo estruturado (Martin et al., 2013).

A dimensão global do uso do smartphone e o seu impacto na vida quotidiana transparecem na emergência de dois novos vocábulos construídos para dar significado às (novas) experiências e comportamentos associados ao seu uso.

Nomofobia é definida como "o medo de estar fora do contato de um telefone móvel" (SecurEnvoy, 2012). O termo é uma abreviação da expressão no-mobile-phone phobia e foi usado pela primeira vez num estudo realizado em 2010 pelos Serviços Postais do Reino Unido para investigar a ansiedade de que sofrem os usuários do telefone móvel (SecurEnvoy, 2012), tendo encontrado cerca de $53 \%$ de utilizadores que se sentiam ansiosos quando perdiam o telemóvel, ficavam sem bateria, sem saldo, ou sem cobertura de rede.

O segundo termo que nasce neste domínio é o Phubbing (sem tradução para o português) e foi um vocábulo cunhado no âmbito de uma campanha publicitária encomendada em 2012 pela Macquarie Dictionary a partir das palavras snubbing e phone para descrever o comportamento de ignorar alguém usando um telefonema, mensagem ou outros através de um smartphone. O termo tem aparecido nos meios de comunicação em todo o mundo, e foi popularizado pela campanha Stop Phubbing criada pela agência McCann (Karadag, et al., (2015) Devido às características dos smartphones o phubbing já é apresentado como um distúrbio que está na intersecção de varias adições assumindo uma estrutura multidimensional: a adição ao smartphone enquanto dispositivo; a adição à internet; a adição às redes sociais e a adição ao jogo (Karadag et al., 2015). Griffiths (2000) parece ter antecipado esta dimensão quando referia que as recentes tendências das TIC encorajam as pessoas a gastar mais tempo com os dispositivos e menos com os seus pares humanos. 
Estamos, pois, perante um fenómeno global, em termos geográficos, mas também em termos do curso de vida pois a utilização do smartphone começa cedo, a par de outras TIC.

De acordo com o estudo Net Children Go Mobile 2013-14 (Simões, Ponte, Ferreira, Doretto, \& Azevedo, 2014), Portugal é líder europeu no acesso à internet por parte de crianças e jovens principalmente através do computador portátil em casa $(65 \%)$, que é seguido do smartphones e dos telemóveis fora de casa, com uso de acesso diário á net por smartphone de $35 \%$. Quanto à idade do primeiro acesso, as crianças mais novas são as que se iniciaram mais cedo no acesso à internet, e as que possuem mais cedo telemóvel e smartphone pessoal. Este dispositivo enquadra-se nas suas rotinas por volta dos 9-10 anos, e é o meio mais referido no acesso diário á internet na escola (22\%), ultrapassando o uso do portátil (14\%). Dois terços dos jovens internautas dizem que nunca se sentiram aborrecidos porque não podiam navegar na internet; contudo, 59\% dos que possuem smartphone refere que muitas ou algumas vezes sentiu uma grande necessidade de verificar o telemóvel para ver se alguma coisa tinha acontecido; $54 \%$ dos possuidores de smartphone refere também que ficou aborrecido quando não podia usar o aparelho porque estava sem rede ou sem bateria (Simões et al., 2014).

De acordo com Massimini e Patterson (2009) é nos jovens adultos e nos estudantes universitários em particular, que se verifica mais intensamente o fascínio pelas TIC. Os smartphones são vistos como factores de relacionamento social e de manutenção das exigências da vida diária, de tal forma que vários investigadores defendem que o uso dos media se tornou uma parte tão significativa da vida dos estudantes que se assume como uma adição invisível, acerca da qual os seus detentores não têm uma noção da verdadeira dimensão da dependência (Roberts, et al., 2014).

Neste enquadramento, o presente estudo teve como objetivo avaliar a existência de padrões de uso do smartphone potencialmente disfuncionais em adultos portugueses.

\section{Método}

\section{Participantes}

Participou no estudo uma amostra de conveniência constituída por 164 participantes, com idades compreendidas entre 18 e 63 anos $(M=29.09$; $D P=10.15$ ); sendo que $7.9 \%$ apresentava como nível de escolaridade o ensino secundário, $41.5 \%$ o $1^{\circ}$ ciclo do ensino superior, $43.3 \%$ o $2^{\circ}$ ciclo do ensino superior e $7.3 \%$ o $3^{\circ}$ ciclo do ensino superior. Entre os participantes, $66.9 \%$ encontrava-se a frequentar o ensino superior no momento em que participaram no presente estudo, $33.65 \%$ dos quais no domínio das ciências da saúde, $55.77 \%$ no domínio das ciências humanas e sociais e $10.58 \%$ no domínio da ciência e tecnologia. Todos os participantes tinham usado smartphone nos últimos 3 meses, apontando como principais razões para o seu uso a comunicação (62.2\%), acesso às redes sociais (26.2\%), pesquisa de informação (8.5\%) e jogos (.6\%). A amplitude da idade com que começaram a usar smartphone foi grande, variando entre os 8 e os 55 anos $(\mathrm{M}=23.13$; $\mathrm{DP}=9.88)$.

\section{Instrumentos}

a) Questionário sociodemográfico: foi desenvolvido especificamente para o presente estudo com objetivo de recolher dados relativos a: sexo; idade; escolaridade; se se encontrava no momento a frequentar o ensino superior e, se sim, em que curso; com que idade começou a usar o smartphone; se o usou nos últimos 3 meses e quais os principais usos que dele faz.

b) Questionário de avaliação de sintomas clínicos sugestivos de uso problemático e de dependência de smartphones: este questionário foi desenvolvido especificamente para o presente estudo, sendo constituído por 10 itens elaborados a partir dos critérios de adicção ao jogo única situação compreendida no capítulo dos comportamentos adictivos sem substância propostos na DSM 5, e que foram ajustados ao uso dos smartphones e que se propõem avaliar: (1) necessidade crescente de usar o smartphone; (2) esforços mal sucedidos para controlar, reduzir ou parar de usar o smartphone; (3) usar o smartphone para diminuir sensação de mal-estar; (4) dissimular a extensão do envolvimento com o smartphone; (5) o uso do smartphone prejudicar relações significativas; (6) não conseguir suportar os custos do uso do smartphone. $\mathrm{O}$ instrumento oferece uma escala de resposta de natureza nominal dicotómica (Sim/Não). O cálculo do score final resulta de um somatório simples das respostas aos itens. Com base em referenciais teóricos (American Psychiatric Association, 2013), e tendo em conta o número de itens deste instrumento, foram assumidos os seguintes pontos de corte para este questionário: com valores entre 0 e 2, considera-se que não existe um uso problemático do smartphone; com valores entre 3 e 4 considera-se que são sugestivos de sintomas moderados de perturbação de uso de smartphones; e quando os valores são iguais ou superiores a 5, considera-se que são sugestivos de sintomas graves de perturbação de uso dos smartphones.

c) Smartphone Addiction Inventory (SPAI) instrumento construído por Lin, et al. (2014), constituindo um questionário de autoavaliação baseado nas características particulares dos smartphones, composto por 26 itens, organizados em quatro fatores: comportamento compulsivo, limitação funcional, abstinência e tolerância. Foi inicialmente testado junto da população de jovens universitários de Taiwan, tendo revelado possuir boa estabilidade temporal e uma excelente consistência interna, bem como uma validade aceitável. A estrutura do SPAI vai ao encontro da taxonomia adotada pelo Diagnostic and Statistical Manual of Mental Disorders da American Psychiatric Association (Lin et al., 2014) para as adições.

\section{Procedimento}

Foi obtido parecer positivo por parte da Comissão de Ética da Universidade Fernando Pessoa e antes de iniciar a recolha de dados todos os participantes deram a sua autorização de forma livre e esclarecida antes do preenchimento dos questionários. Não foram recolhidos 
dados pessoais dos participantes que os permitissem identificar. Foram definidas como condições de inclusão na amostra serem indivíduos da população portuguesa, maiores de idade e capazes de dar o seu consentimento informado de forma livre. A administração dos questionários foi realizada por via eletrónica (on-line), tendo sido efetuado um convite à participação no estudo através das redes sociais, e os dados foram automaticamente importados para uma base de dados eletrónica em EXCEL, posteriormente convertida para análise através do programa SPSS.

\section{Resultados}

Os resultados revelam que todos os participantes utilizaram smartphone nos últimos 3 meses e que o principal uso que lhes tê dado se encontra relacionado com a comunicação $(62.2 \%)$.

A idade de início desse uso de smartphone varia entre os 8 e os 55 anos $(M=23.13 ; D P=9.88)$.

A análise de dados sugere que $90.9 \%(n=149)$ dos participantes não apresenta sintomas sugestivos de perturbação associada ao uso de smartphones, 6.7\% $(n=11)$ apresenta sintomas moderados de perturbação de uso de smartphones e $2.4 \%(n=4)$ apresenta sintomas graves de perturbação de uso.

No Quadro 1, apresentam-se os resultados das análises descritivas conduzidas e que nos permite constatar que, de forma geral, os participantes apresentam valores baixos no que respeita aos sintomas de adição aos smartphones em geral e aos sintomas de compulsão, limitação funcional, abstinência e tolerância.

Quadro 1.

Análise descritiva dos valores obtidos no inventário globalmente considerado e nas suas subescalas

\begin{tabular}{lccccc}
\hline $\begin{array}{l}\text { SPAI [valor mínimo possível } \\
\text { - valor máximo possível] }\end{array}$ & Min. & Max. & Med & $M$ & DP \\
\hline Comportamento Compulsivo & 9 & 24 & 12 & 12.85 & 3.77 \\
[9-36] & & & 9 & 10.29 & 3.22 \\
Limitação Funcional [8-32] & 8 & 24 & 9 & 9.29 & 3.04 \\
Abstinência [6-24] & 6 & 20 & 8.5 & 9.62 & 1.90 \\
Tolerância [3-12] & 3 & 12 & 5 & 5.62 \\
Total [26-104] & 26 & 77 & 35 & 38.07 & 10.37 \\
\hline
\end{tabular}

Quando comparados participantes que apresentam sintomas graves de perturbação de uso de smartphones com os que apresentam sintomas moderados de perturbação de uso de smartphones e com aqueles que não apresentam sintomas de dependência, verificou-se que os participantes que apresentam valores mais elevados ao nível do Comportamento Compulsivo $(F=28.01 ; p<.0001)$, Limitação Funcional $(F=55.23$; $p<.0001)$, Abstinência $(F=26.07 ; p<.0001)$, Tolerância $(F=7.18 ; p<.01)$ e um score global no SPAI $(F=40.48$; $p<.0001)$ mais elevado são os que apresentam sintomas graves de dependência dos smartphones, seguidos pelos participantes que apresentam sintomas moderados deste tipo de perturbação e, por último, dos participantes que não apresentam sintomas sugestivos de perturbação associada a esse uso (Quadro 2).
Quadro 2.

Análise comparativa entre participantes com diferentes níveis de sintomas de dependência de smartphones quanto aos valores obtidos no SPAI globalmente considerado e nas suas subescalas

\begin{tabular}{lccccc}
\hline \multicolumn{1}{c}{ Nivel de } & $C$. & $L$. & Abstin. & & SPAI \\
Dependência & Compul. & Func. & & Tolerân. & Total \\
& $M$ & $M$ & $M$ & $M$ & $M$ \\
& $D P$ & $D P$ & $D P$ & $D P$ & $D P$ \\
\hline $\begin{array}{l}\text { Sem sintomas } \\
\text { de dependência }\end{array}$ & 12.31 & 9.76 & 8.86 & 5.48 & 36.41 \\
$\begin{array}{l}(n=149) \\
\text { Sintomas }\end{array}$ & 3.25 & 2.40 & 2.64 & 1.80 & 8.35 \\
moderados & 16.45 & 13.27 & 12.36 & 6.36 & 48.45 \\
$(n=11)$ & 3.86 & 3.69 & 3.01 & 2.06 & 10.74 \\
$\begin{array}{l}\text { Sintomas graves } \\
(n=4)\end{array}$ & 23.00 & 22.00 & 17.00 & 8.75 & 70.75 \\
& 2.00 & 2.16 & 2.45 & 2.50 & 8.02 \\
\hline
\end{tabular}

Legenda: C. Compul. - Comportamento Compulsivo; L. Func. - Limitação Funcional; Abstin. - Abstinência; Tolerân. Tolerância

\section{Discussão}

A análise dos dados sugere que a maior parte dos participantes do presente estudo não apresenta sintomas sugestivos de perturbação associada ao uso de smartphones, ainda que se sinalize $9.1 \%$ de adultos que terão um uso potencialmente disfuncional do mesmo, com apresentação de comportamentos compulsivos, sintomas de abstinência e tolerância, bem como com consequências desse uso em termos de limitação funcional. Estes resultados são ligeiramente inferiores aos descritos na literatura, que apontam para que, entre jovens adultos, a dependência do smartphone esteja presente em $15.38 \%$ destes (Lapointe, Boudreau-Pinsonneault, \& Vaghefi, 2013). Ainda que a reduzida dimensão da amostra estudada nos leve a considerar estes resultados com reservas, consideramos que estes vêm sublinhar a importância da realização de rastreios no domínio do uso problemático e da adição de smartphones na população adulta portuguesa, ponto de partida, por um lado, para a sensibilização e psicoeducação da população relativamente a usos potencialmente disfuncionais, e, por outro, para o encaminhamento para intervenção psicológica.

\section{Referências}

American Psychiatric Association. (2013). DSM 5Manual de Diagnóstico e Estatística das Perturbações Mentais ( $5^{\mathrm{a}}$ Ed.) Lisboa: Climepsi Editores.

Echeburúa, E., \& Corral, P. (2010). Adicción a las nuevas tecnologías y a las redes sociales en jóvenes: Un nuevo reto. Adicciones, 22 (2), 91-95.

Grant, J.E., Potenza, M.N., Weinstein, A., \& Gorelick, D.A. (2010). Introduction to behavioral addictions. The American Journal of Drug and Alcohol Abuse, 36(5), 233-241.http://doi.org/10.3109 /00952990.2010.491884

Griffiths, M. (1996). Behavioural addiction: An issue for everybody? Employee counselling today. The Journal of Workplace Learning, 8(3), 19-25. 
Griffiths, M.D. (1995). Technological addictions. Clinical Psychology Forum, 14-19.

Griffiths, M.D. (1999). Internet addiction: Fact or fiction? The Psychologist: Bulletin of the British Psychology Society, 12, 246-250.

Griffiths, M.D. (2000). Does Internet and computer "addiction" exist? Some case study evidence. CyberPsychology \& Behavior, 3(2), 211-218.

Grover, A., Kamins, M.A., Martin, I.M., Davis, S., Haws, K., Mirabito, A.M., ...Rapp, J. (2011). From use to abuse: When everyday consumption behaviors morph into addictive consumptive behaviors. Journal of Research for Consumers, 19, 1-8.

Holden, C. (2010). Behavioral addictions debut in proposed DSM-V. Science, 327(5968), 935-935. http://dx.doi.org/10.1126/science.327.5968.935.

International Telecommunication Union (2015) http://www.itu.int/net/pressoffice/press_releases/2015 /17.aspx\#.WWPy-MaZPeR

Internet World Stats, 2017 http://www.internetworldstats.com/stats.htm

Karadag, E., Tosuntas, S.B., Erzen, E., Duru, P., Bostan, N., Sahin, B.M., ... Babadag, B. (2015). Determinants of phubbing, which is the sum of many virtual addictions: A structural equation model. Journal of Behavioral Addictions, 4(2), 60-74.

Kwon, M., Kim, D. J., Cho, H., \& Yang, S. (2013). The smartphone addiction scale: Development and validation of a short version for adolescents. PloS One, 8(12), e83558. doi:https://doi.org/10.1371/journal.pone.0083558

Lapointe, L., Boudreau-Pinsonneault, C., \& Vaghefi, I. (2013). Is smartphone usage truly smart? A qualitative investigation of IT addictive behaviours. In Hawaii International Conference on System Sciences. doi: 10.1109/HICSS.2013.367

Levitin, D.J. (2015). The organized mind: Thinking straight in the age of information overload. New York: A Plume Book.

Lin, Y.-H., Chang, L.-R., Lee, Y.-H., Tseng, H.-W., Kuo, T.B.J., Chen, S.-H. (2014). Development and validation of the Smartphone Addiction Inventory (SPAI). PLoS ONE 9(6):e98312. doi:http://dx.doi.org/10.1371/journal.pone.0098312

Martin, I.M., Kamins, M.A., Pirouz, D.M., Davis, S.W., Haws, K.L., Mirabito, A.M., ... Grover, A. (2013). On the road to addiction: The facilitative and preventive roles of marketing cues. Journal of Business Research, 66, 1219-1226.

Massimini, M., \& Peterson, M. (2009). Information and communication technology: Affects on U.S. college students. CyberPschology: Journal of Psychosocial Research on Cyberspace, 3(1), 1-12.

Orford, J. (2008). Asking the right questions in the right way: the need for a shift in research on psychological treatments for addiction. Addiction,103 (6), 875-885. doi: 10.1111/j.1360-0443.2007.02092.x.

Oulasvirta, A., Rattenbury, T., Ma, L., \& Raita, E. (2012). Habits make smartphone use more pervasive. Personal and Ubiquitous Computing, 16(1), 105-114. http://dx.doi.org/10.1007/s00779-011-0412-2.
Pinna, F., Dell'Osso, B., Di Nicola, M., Janiri, L., Altamur, A.C., Carpiniello, B., \& Hollander, E. (2015). Behavioural addictions and the transition from DSM-IV-TR to DSM-5. Journal of Psychopatholology, 21(4), 380-389.

Rivas, M., Fernandez, L., \& Guadix, M. (2009). Adicción y abuso del teléfono móvil. In $\mathrm{E}$. Odriozola, F. Labrador, \& E. Iglesias (Coords.), Adicción a las nuevas tecnologías en adolescentes y jóvenes (pp. 131-150). Madrid: Edições Pirámide.

Roberts, J. A., Yaya, L. H. P., \& Manolis, C. (2014). The invisible addiction: Cell-phone activities and addiction among male and female college students. Journal of Behavioral Addictions, 3(4), 254-265. doi:10.1556/JBA.3.2014.015.

Rosenberg, K., \& Feder, L. (2014). An introduction to behavioral addictions. In K. Rosenberg, \& L. Feder (Eds.), Behavioral addictions, criteria, evidence, and treatment (pp. 1-17). New York, NY: Elsevier. doi:10.1016/B978-0-12-407724-9.00001-X.

Salehan, M., \& Negahban, A. (2013). Social networking on smartphones: When mobile phones become addictive. Computers in Human Behavior, 29(6), 2632-2639.

http://dx.doi.org/10.1016/j.chb.2013.07.003

SecurEnvoy (2012). 66\% of the population suffer from Nomophobia the fear of being without their phone. Retirado a 12 de Maio de 2014 de http://www.securenvoy.com/blog/2012/02/16/66-of-th e-population-suffer-fromnomophobia-the-fear-of-bein g-without-their-phone/.

Shambare, R., Rugimbana, R., \& Zhowa, T. (2012). Are mobile phones the 21 st century addiction? African Journal of Business Management, 62(2), 573-577.

Simões J. A., Ponte, C., Ferreira, E., Doretto, J., \& Azevedo, C. (2014). Crianças e meios digitais móveis em Portugal: Resultados nacionais do Projeto Net Children Go Mobile. Lisboa: Centro de Estudos de Sociologia da Universidade Nova de Lisboa.

Sussman, S., Leventhal, A., Bluthenthal, R. N., Freimuth, M., Forster, M., \& Ames, S. L. (2011). A framework for the specificity of addictions. International Journal of Environmental Research and Public Health, 8(8), 3399-3415. http://doi.org/10.3390/ijerph8083399 .

Turel, O., Serenko, A., \& Giles, P. (2011). Integrating technology addiction and use: An empirical investigation of online auction users. MIS Quarterly, 35(4), 1043-1062. 